\title{
Políticas Públicas para la Agricultura Familiar en Colombia: Contribuciones y Retos para el Desarrollo Rural
}

\author{
Yamile Lined Ramos Mahecha ${ }^{1 *}$, \\ Programa de Pós-Graduação em Desenvolvimento Territorial e Políticas Públicas, da PPGDT/ \\ Universidade Federal Rural do Rio de Janeiro, Seropédica - RJ, Brasil
}

Recebido em 24 de Julho de 2016. Aceito em 22 de Agosto de 2016 RESUMO

$\mathrm{O}$ artigo discute o processo e desenvolvimento da agricultura familiar na Colômbia e suas políticas públicas. O objetivo deste trabalho é apresentar uma visão geral por meio de referencias bibliográfica, documental, informes e eventos com relação com o assunto. Destina-se a conhecer e aprender mais sobre o assunto, porque é de extrema importância tanto nacional como internacionalmente. A Colômbia segue a tendência do que aconteceu nos países desenvolvidos, o aumento da participação da agricultura no PIB é mais baixo, em consideração com outros sectores, como a indústria e os serviços. Mesmo assim não deixa de ser importante em relação a sua contribuição à economia e ao desenvolvimento social. Uma das questões mais criticadas e gera muitas perguntas é a questão da produção de estatísticas relevantes, que sejam oportunas para corrigir as diferenças do modelo, que tem pontos fracos. Um modelo integrado, onde tudo documentado e dados, processa a coisa mais importante é facilmente acessível para pessoas que precisam deste tipo de informações, onde podemos mostrar a evolução de cada etapa do projeto, monitoramento e avaliação das políticas públicas.

Palavras-chave: agricultura familiar; políticas públicas; desenvolvimento rural. ABSTRACT

The article discusses the process and development of family farming in Colombia and its public policies. The aim of this paper is to present an overview through bibliographical, documentary, reports and events related with the subject. This article aims to know and learn more about the issue because it is of paramount importance both nationally and internationally. Colombia follows the trend of what happened in developed countries, increasing the share of agriculture in GDP is lower, in consideration with other sectors such as industry and services. Even so it is not without importance in relation to the contribution of the economy and social development, where its role is highlighted. One of the most criticized issues and generates many questions is the issue of the production of relevant statistics to correct those differences model, which has weaknesses. An integrated model where all documented and data, processes the most important thing is easily accessible for people who need this type of information, where we can show the evolution of each step of design, monitoring and evaluation are each public policy.

Keywords: family farming; public policy; rural development.

\section{RESUMEN}

El artículo aborda el proceso y desarrollo de la agricultura familiar en Colombia y sus políticas públicas. El objetivo de este artículo es presentar un panorama general por medio de referencias bibliográficas, documentales, informes y de algunos eventos relaciones con el tema. Se pretende saber y conocer más sobre el asunto, ya que es de suma importancia tanto a nivel nacional como internacional. Colombia sigue la tendencia de lo que ha ocurrido en los países desarrollados, cada vez la participación del sector agropecuario en el PIB es menor, en consideración con otros sectores como la industria y los servicios. Mismo así no deja de tener importancia en relación al aporte de la economía y desarrollo social, donde se destaca su papel. Una de las cuestiones más criticadas y que genera muchos cuestionamientos es el tema de la producción de estadísticas relevantes, que corrijan aquellas divergencias, que presenta debilidades en la oferta de las estadísticas. Un modelo integrado en donde se encuentren todos los datos documentados, procesos y lo más importante que sea de fácil acceso para las personas que necesitan este tipo de información, donde se pueda evidenciar la evolución de cada paso de diseño, seguimiento y evaluación de cada una de las políticas públicas.

Palabras Clave: Agricultura familiar; Políticas públicas; Desarrollo rural.

1. Mestre em Políticas Publicas e Desenvolvimento Territorial pela Universidade Federal Rural do Rio de Janeiro

* Autor para correspondência. Yamile Lined Ramos Mahecha

e-mail: lined.ramos@gmail.com 


\section{Introducción}

Este trabajo pretende dar a conocer el proceso y desarrollo de la agricultura familiar en Colombia, como también algunas de sus más importantes políticas públicas, resaltando a labor tan importante que desempeñan para el sector rural y para el desarrollo sustentable de cada país. El sector mismo aportando para la agricultura el $80 \%$ de la producción del café y una tercera parte con relación a la producción pecuaria, se encuentra amenazado por numerosos factores, como acuerdos comerciales, macroeconómico, y uno de los principales que es el conflicto armado que se vive en áreas rurales y que desestabiliza los sistemas productivos.

Al igual que muchos países de América Latina, Colombia se ha arriesgado por un modelo económico que continua favoreciendo a los grandes empresarios agrícolas en comparación con los pequeños productores, los cuales son beneficiarios de políticas asistencialistas que no favorecen un desarrollo integral y sostenido para o sector rural, más específicamente para los agricultores familiares.

Así mismo no ha habido una política por parte del estado consolidada, muy por el contrario cada uno de los gobiernos que asume el poder llega con propuestas que no se articulan con las existentes, entonces no existe una continuidad a los procesos ni a las políticas creadas a favor del sector, llegando a ser acciones desarticuladas, fragmentadas y coyunturales que no abarcan la situación de fondo.

Por otro lado los movimientos sociales no se encuentran organizados y tienen poca fuerza del sector, aunque recientemente se ha visto una mayor movilización del campesinado, no se encuentra totalmente consolidado ni con la fuerza necesaria para crear presión sobre el gobierno y exigir sus derechos. Otro tema de suma importancia que afecta el sector rural es la pertenencia de las tierras, para esa situación se han realizado una serie de reformas que hasta la actualidad no se ha logrado una solución concreta, teniendo en cuenta que es un poco más difícil por el tema de la violencia y la desapropiación de tierras, para esto se creó en el Ministerio de Agricultura y desarrollo rural (MADR), una unidad de restitución de las tierras. En el presente artículo se van a dar a conocer algunas políticas del MADR y otras organizaciones del gobierno que contribuyen en el tema y que de alguna manera inciden en las dinámicas de los agricultores familiares.

Este artículo aborda tres cuestiones, la primera que hace referencia a un breve contexto de la agricultura familiar en Colombia. En segundo lugar se abarcan las políticas públicas para la agricultura familiar y el sector rural y en la tercera y última parte se expone los impactos generales de las políticas públicas y el papel de la agricultura familiar en el desarrollo rural. Estos pontos fueron desarrollados con base en informaciones segundarias partiendo de una revisión de la literatura y de diferentes publicaciones como artículos, eventos, informes, documentos y libros entre otros.

\section{Metodología}

Este artículo es resultado de una pesquisa documental a partir de un interés por el tema. Por medio del acceso a diferentes documentos, artículos sobre eventos, informes y libros, entre otros. Se trata de sintetizar y dar una mirada general a un tema que en los últimos años ha tomado mayor fuerza, significado y se ha conseguido con que cada vez más, exista una grande preocupación en cuestionar, criar y desenvolver políticas que favorezcan de manera eficaz y eficiente a la populación rural y con un foco relevante para la agricultura familiar.

Una vez que se investigó algunos informes, textos y libros publicados y relacionados con el tema, se llevó a cabo la selección de aquellos que podrían contribuir de manera significativa para el desarrollo del presente trabajo dando de manera general un panorama de un tema que por decirlo de alguna manera se encuentra de moda en los últimos años, y que ha venido generando un proceso lento más cada vez mejor consolidado de debates sobre el asunto, se pretende que con el recurrir del tiempo tome mayor fuerza,

Políticas Públicas para la Agricultura Familiar en Colombia: Contribuciones y Retos para el Desarrollo Rural Yamile Lined Ramos Mahecha, María Isabel Saz Gil, Ana Isabel Zardoya Alegría e

Angela Serafim Godinho Espíndula 
preocupación y critica para proponer, criar y desarrollar políticas que contribuyan de manera eficaz y eficiente al desarrollo rural del país.

\section{Génesis y desarrollo del concepto de la Agricultura Familiar}

Las orígenes del concepto de la agricultura familiar en América Latina emergieran en las ideas populistas de finales del siglo XIX y principios del XX, que tenía el campesinado europeo en un elemento de estabilidad y cohesión social (MALETTA, 2011). De acuerdo con Alfa y Castellán (2008), hay varios estudios sobre el mundo campesino europeo, donde se reconoce al campesinado como una clase marcada por un fuerte vínculo entre su país, su territorio, los grupos sociales pertenecientes a estos territorios, sus culturas y reglas que son propios de ese espacio.

A principios del siglo XX un ruso llamado Alexander Chayanov, creador de la teoría de la unidad económica campesina, argumento que la economía campesina tiene un carácter familiar, e indica que la organización de la economía campesina se determina por la "composición de la familia campesina, la coordinación, demandas de los consumidores, así como el número de trabajadores que tienen "(Wolf, 1982, p. 25).

El mundo comenzó a reconocer que la unidad económica campesina tenía características sociales y económicas específicas a partir de los trabajos de Chayanov. De este modo, Hernández (1993) señala que la actividad económica de la empresa rural está impulsado por la necesidad de la unidad de producción de subsistencia. Es decir, a diferencia de las empresas capitalistas donde hay un intercambio, la agricultura familiar utiliza su propia producción.

Según Sánchez (2011), el concepto de la agricultura familiar en los países desarrollados se creó durante la primera mitad del siglo XX, ya que el sector se compone de actividades en las que la mano de obra familiar es predominante. Campos y Garner (2012) también señalan que la definición anterior de la agricultura familiar surge en los Estados Unidos con el trabajo de Johnson (1944), este trabajo, argumentó que la agricultura familiar se caracteriza por la mano fuerte de la adicción el trabajo familiar.

En el siglo XX, el concepto más utilizado era el campesinado que, en la década de 1960, se caracterizó como un sector social históricamente sometidos a situaciones de desigualdad y pobreza, en gran parte por la estructura de propiedad de la tierra existente en la mayoría de los países de América Latina, marcada por un modelo latitudinario, que representó la mayor proporción de tierra cultivable. Con el fin de poner de relieve la existencia de una estructura agraria dicotómica y desigual, el concepto de campesino era equivalente a los pequeños agricultores, lo que condujo a un proceso de reforma agraria en varios países de América Latina, lo que sin duda contribuyó a satisfacer sector y su racionalidad económica y social (SALCEDO e GUZMAN, 2014).

En los años 1980 y 1990, la agricultura familiar, también llamado el sector campesino, pasó desapercibida en América Latina y el Caribe. El sistema económico de estos países se definió por la globalización y la agroindustria, en concreto el modelo de exportación, desarrollo e implementación de políticas y programas que permiten se incluyen los agricultores familiares.

Para Salcedo y Guzmán (2014), en América Latina y el Caribe, el término "agricultura familiar 'fue reconocido oficialmente en 2004 con la creación de la Reunión Especializada sobre Agricultura Familiar (REAF), en la que los miembros del MERCOSUR y la REAF miembros establecieron y construyeron algunos parámetros o criterios generales para la definición de la agricultura familiar, se aplica de acuerdo a la realidad de cada país (Argentina, Brasil, Paraguay y Uruguay). Con el único propósito de promover el reconocimiento mutuo de los agricultores familiares en cada país, la resolución del Grupo Mercado Común (GMC), el máximo órgano ejecutivo del MERCOSUR, reconoce la existencia, validez y la importancia de la AF y el establecimiento de criterios para identificar. 
El GMC ha establecido los siguientes criterios comunes para la identificación de las poblaciones rurales que componen toda la agricultura familiar: a) la mano de obra empleada en el establecimiento deberá ser predominantemente familiar, con escasa presencia de trabajadores contratados; b) la familia es directamente responsable de la producción y gestión de las actividades agrícolas y debe residir en o en una localidad próxima; c) los recursos productivos utilizados son compatibles con la capacidad de la mano de obra familiar, con la actividad y la tecnología utilizada, de acuerdo a la realidad de cada país (que es una relación directa entre la capacidad de los agricultores y el uso de los recursos) .

Además, los hombres y las mujeres que son agricultores sin tierra, los beneficiarios de los procesos o programas de reforma agraria para acceder y permanecer en la tierra, así como las comunidades de productores que hacen uso común de la tierra, que forma parte de la agricultura familiar, a condición de que se cumplen los criterios mencionados anteriormente.

Esta comprensión de la definición de la agricultura familiar permitió un mejor posicionamiento del sector con las agendas de los gobiernos, por el reconocimiento de las contribuciones de la AF para el desarrollo rural sostenible (SALCEDO y GUZMÁN, 2014). Según Schneider (2012), en los últimos años, el concepto de la AF está en camino de ser una categoría política emergente en algunos países de América Latina y el Caribe, con el aumento de legitimidad social, lo que resulta en la definición de políticas y estrategias públicas dirigidas especialmente al desarrollo del sector.

\section{Contexto de la agricultura familiar em Colombia}

Dadas las condiciones geográficas en las que se encuentra ubicada Colombia, con una ventaja en su localización intertropical y ecuatorial, es un país que tiene un grande potencial productivo si se utilizaran esas ventajas para asegurar la demanda de alimentos del país, que eso se refleja en ganancias vinculadas a exportación de productos que son competitivos internacionalmente. Segundo un estudio de la FAO que se encarga de evaluar el potencial de expansión del área agrícola en el mundo, Colombia se ubica en el puesto 25 entre 223 países, eso deja ver que el país es una de las naciones con mayor potencial de expansión de tierras para el uso agrícola y también con una disponibilidad de recursos hídricos consideradas las más altas del mundo. (Vélez, et al., 2010).

El país dispone de suficientes recursos naturales como suelo y tierras que solo se articula bien mediante la planificación y el ordenamiento territorial del país, se puede llegar a consolidar a seguridad alimentaria y producir de manera satisfactoria recursos para la nación. (Malagón, 2002).

El sector económico de la agricultura se encuentra en el tercer lugar, ya que es el generador de los empleos en Colombia. Según cifras oficiales del Instituto Geográfico Agustín Codazzi (IGAC, 2012), en términos generales se dice que únicamente se emplea el $24,1 \%$ del potencial agrícola Colombiano y que no en tanto el potencial agropecuario del país se aproxima al 36,2\% del territorio, que es comprendido por sistemas tradicionales, donde está la agricultura con 19,3\%, seguida de la ganadería con 13,3\%. (Perfetti y Cortés, 2013).

Según Gutiérrez (2009). Con relación al PIB agrícola Colombiano, con el recurrir de los años continua perdiendo participación, en 1970 se contaba con el 25,33\%, ya en el 2007 paso a 11,44\% y continuo bajando para el 2008 con 8,5\%, todavía así no deja de ser importante para la agroindustria y el comercio exterior, como también se encuentra asociada a los aspectos del medio ambiente, a generación de empleo y la calidad de vida de las personas. La participación del sector dentro del PIB nacional se encuentra en la quinta posición, y su promedio de participación es del 9,2\% entre los anos de 2000-2008. Manteniéndose con una tasa de crecimiento del 3,0\% en los últimos 9 anos.

Por otra parte lo que se refiere a populación, Colombia tiene un número significativo de personas que viven en áreas rurales, según el Departamento Administrativo Nacional de Estadística (DANE) en el 2012 se tenía 11.204 .685 personas, con actividad agrícola como a principal fuente de sustento. CRECE, 2006 y Perfetti, 2009. Desde el punto de vista político para Machado y Botello (2013), ese debate en la actualidad de la cuestión rural-agrícola en Colombia, está planteado desde un modelo de desarrollo rural basado por un lado por la pequeña propiedad y producción y por otro lado por un modelo empresarial de grandes empresas modernas relacionadas con el estado y la política pública. 
Para Machado (2009) el tema agrario en Colombia ha estado caracterizado históricamente por problemas con el acceso a la propiedad por parte de los pequeños y medianos productores, siendo evidenciado la debilidad de los derechos de la propiedad y a imposibilidad del estado para no garantir estos. Este tema genera malestar y problemas entre actores como los propietarios y campesinos, y estés con la sociedad rural, urbana y el Estado, estos conflictos viene desde la época colonial hasta el día de hoy.

Colombia desde los años sesenta ha vivenciado un proceso de reforma agraria, sin tener mayor repercusión y sin tener resultados con lo referente a la tenencia de la tierra. Para Gómez (2011) esa reforma (Ley 135 de 1961, Ley $1^{a}$ de 1968) fue marginal y redistribucionista que hizo una separación superficial de la estructura agraria. Con la Ley $4^{\text {a }}$ de 1973 se estancaron los procesos de reforma agraria, ya con la Ley 35 de 1982 hubo una agilidad en los trámites que concierne a entrega de las tierras en zonas de conflicto. Más adelante con la Ley 30 de 1988 se dieron cambios significativos en el proceso de reforma agraria, mismo con las leyes anteriores se continuaba siendo marginal.

Más adelante se crea el Sistema Nacional de Reforma Agraria y Desarrollo Rural Campesino con la Ley 160 de 1994, que establece un subsidio para la adquisición de tierras, del mismo modo se reforma el Instituto Colombiano de Reforma Agraria (INCORA), con esta Ley se entiende por Unidad Agrícola Familiar (UAF) como la empresa básica de producción pecuaria, agrícola o forestal, cuya extensión es según las condiciones agroecológicas y con apropiada tecnología, permiten a la familia una remuneración por su trabajo y contribuye con la formación de su patrimonio. Sabourin, et al., (2014)

No se es posible identificar desde el punto de vista de referencia del desarrollo rural en Colombia el concepto de "agricultor familiar", por el contrario de hace referencia al concepto de como "pequeño y mediano productor", en ese caso con relación al número de UAF que se tiene. Lo que contempla la Ley es que el concepto está definido de acuerdo con el tamaño de la propiedad, - Ley 77 de 1987 y 501 de 1989, que es donde se reglamentan decretos, se encuentra relacionado lo que concierne a prestación del servicio de asistencia técnica agropecuario a pequeños productores y aparece la definición del pequeño productor en beneficio de la prestación de servicios de asistencia técnica agropecuaria y se dice que los pequeños productores son los campesinos propietarios poseedores de un título que con ayuda de su familia exploten una propiedad rural y que además no supere dos UAF, además de proveer de su actividad agrícola por lo menos 70\% de sus ingresos. Esto se encuentra contemplado en el artículo 28 del Decreto 1946 de 1989). Sabourin, et al., (2014).

\section{Políticas públicas para la agricultura familiar y el sector rural}

Según el contexto de las políticas públicas en Colombia relacionadas con el tema de la agricultura familiar o pequeño productor campesino como ya vimos, es caracterizado por dificultades que se presentaron históricamente en su enfoque y su institucionalidad que la mantiene, y eso se ve reflejado en la agricultura familiar. El modelo de modernización en el cual se enmarco las políticas, se ha enfatizado más en la cuestión productiva y en la competitividad que en aspectos sociales e institucionales. Actualmente el gobierno tiene una preocupación mayor con tratar de introducir cambios y tendencias que refleje una política menos discriminatoria y excluyente. Este proceso no se ha desarrollado plenamente por cuestiones del acuerdo agrario que resulta de conversaciones de paz con las Fuerzas Armadas Revolucionarias de Colombia (FARC) que son realizadas en La Habana, en lo que se refiere a política y desarrollo rural. Machado et al. (2013)

El principal órgano público encargado de dirigir políticas y programas orientados para el pequeño productor es el Ministerio de agricultura y Desarrollo Rural (MADR), a través de diferentes órganos e entidades prestadoras y vinculadas que hacen parte de la misma como lo son: Banco Agrario, Autoridad Nacional de Acuicultura y Pesca (AUNAP), Corporación Colombiana de Investigación Agropecuaria (CORPOICA), Fondo para el Financiamiento del Sector Agropecuario (FINAGRO), Corporación Colombia Internacional (CCI), Instituto Colombiano de Desarrollo Rural (INCODER), el Instituto Colombiano 
Agropecuario (ICA), Unidad de Restitución de Tierras y la Unidad de Planificación Rural Agropecuaria (UPRA). Adicionalmente a las anteriores instituciones se encuentran otras que auxilian con el desarrollo de programas que benefician a los pequeños productores, como el caso del Departamento para la Prosperidad Social (DPS), el Servicio Nacional de Aprendizaje (SENA).

Machado y Botello (2013), consideran que as reglas institucionales del sector agropecuario (acuerdos, normas, instrumentos de políticas sectoriales, alianzas, entre otras), son orientadas para el sector empresarial. Lo que se dice es que la creencia política de que la pequeña producción no es competitiva y que tanto la población rural como el PIB agropecuario tienden a decrecer. Uno de los aspectos importantes dentro de esta dinámica es que los proyectos promovidos y financiados por entidades internacionales no son totalmente articulados con los órganos públicos encargados de ejecutarlas y es donde está el error y termina por presentarse una fragmentación de las acciones en los territorios.

Maletta (2011), considera que la primordial entidad promotora con relación a la agricultura familiar que se encuentra dentro del gobierno Colombiano es el Instituto Colombiano de Desarrollo Rural (INCODER), que fue creado en el 2003 luego de un ordenamiento por parte del gobierno mediante el decreto 1300 del mismo año, donde ordeno la supresión del INCORA, como también del Fondo de Cofinanciación para la Inversión Rural (DRI), del Instituto Nacional de Adecuación de Tierras (INAT) y del Instituto Nacional de Pesca y Acuicultura (INPA).

El INCODER es la guía de acción e inversión dentro del plan estratégico del mismo, este se encuentra en continua actualización para asegurar la coherencia, los objetivos y las políticas nacionales. En su última actualización que fue en el periodo del 2013-2014 se buscó a profundar más sobre el desarrollo rural con un enfoque territorial. Lo que dentro de este sentido significa promover, ejecutar y coordinar procesos de cambios en lo social, productivo e institucional de los territorios rurales prioritarios, con el fin de mejorar las desigualdades de las familias y las comunidades regionales.

Por otro lado en lo que concierne a la inversión del plan en la gestión del Marco de Gasto de Mediano Plazo (MGMP), es un presupuesto de $\$ 835.881$ para los dos años, que se encuentran distribuidos de la siguiente forma: $\$ 411764$ para el 2013 y $\$ 424117$ para el 2014 y a su vez este repasa para: para generación de activos e ingresos el 37\%, para adecuación de tierras el 28\%, para ordenamiento de la propiedad un 18\%, para ordenamiento de la propiedad de grupos étnicos $14 \%$, y para fortalecimiento institucional el 3\%. Sabourin, et al. (2014)

\section{DISTRIBUCIÓN DEL PRESUPUESTO 2013-2014}

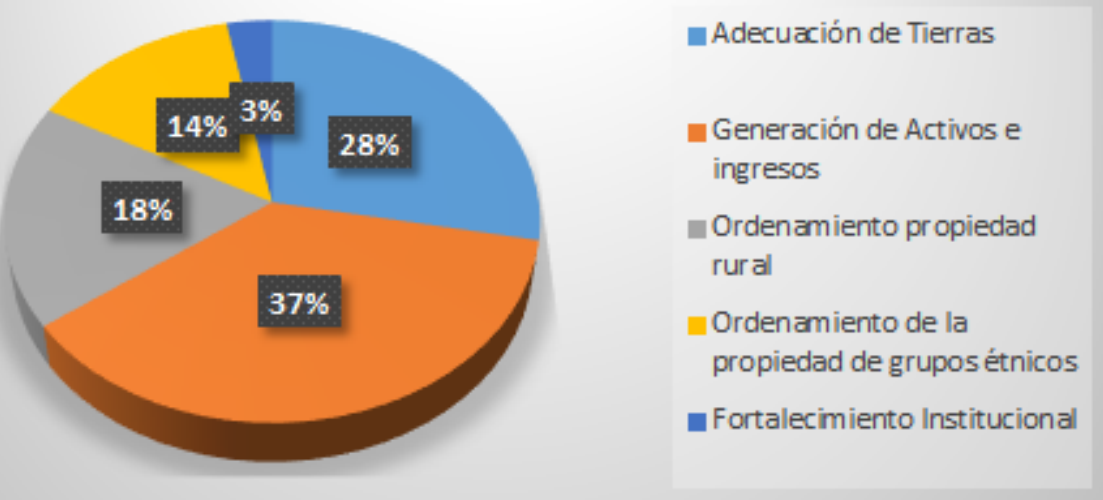

GRÁFICO 1. Distribución del presupuesto de 2013-2014

FUENTE: INCODER, 2013. 
Basado en el Informe de Rendición pública de cuentas en la gestión del 2013-2014, presentado para el Ministerio de Agricultura y Desarrollo Rural (MADR), y encaminado para el Ministro Rubén Darío Lizarralde. A continuación, se sintetiza en una tabla los programas más significativos dentro de las políticas públicas para la agricultura familiar o pequeños productores, como también programas para el desarrollo rural de los territorios, en ella se encuentra distribuido los programas con los principales temas específicos de cada uno y las acciones que soportan estos programas. Tabla No. 1

Tabla No. 1. Agrupamiento de las políticas públicas, programas y acciones vinculados al MADR 


\begin{tabular}{|c|c|c|}
\hline PROGRAMAS & TEMAS & ACCIONES \\
\hline \multirow{11}{*}{ 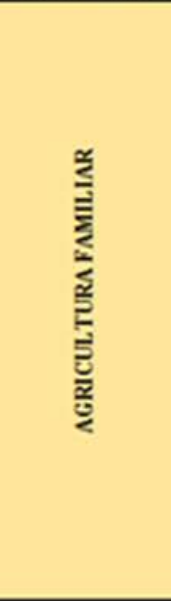 } & Instrumentos financieros & \multirow{11}{*}{$\begin{array}{l}\text { Ministerio de Comercio, Industria y Turismo } \\
\text { La Gobemación } \\
\text { Escuela Superior de Administración Pública - } \\
\text { ESAP } \\
\text { Ministeria de Agricultura y Desarrollo Rural } \\
\text { (MADR) }\end{array}$} \\
\hline & $\begin{array}{l}\text { Apoyo integral a los proyectos productivos y de } \\
\text { innovación }\end{array}$ & \\
\hline & $\begin{array}{l}\text { Recuperación y reproducción de semillas criollas y } \\
\text { nativas }\end{array}$ & \\
\hline & Formalización empresarial & \\
\hline & $\begin{array}{l}\text { Identificación, Conformación y acompaçamiento de } \\
\text { clúster prođuctivos }\end{array}$ & \\
\hline & $\begin{array}{l}\text { Rondas regionales de inversión y innovación em } \\
\text { agricultura familiar }\end{array}$ & \\
\hline & Compras institucionales & \\
\hline & Mercados campesinos & \\
\hline & Fondo de gobemanza campesina & \\
\hline & Fortalecimiento de las secretarias de agricultura & \\
\hline & $\begin{array}{l}\text { Registro Unico Nacional de agricultura familiar } \\
\text { (RUNAF) }\end{array}$ & \\
\hline \multirow{22}{*}{ 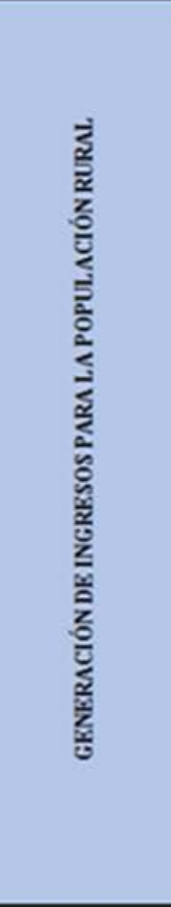 } & Politica integral de tiemas & \multirow{22}{*}{$\begin{array}{l}\text { Unidad de Restitución de Tierras (URT) } \\
\text { Comite Regional de Asignación de Recursos } \\
\text { (CREAR) } \\
\text { FINAGRO } \\
\text { Instituto Colombiano de Desarrollo Social } \\
\text { Incoder }\end{array}$} \\
\hline & Ley de victimas y restitución de tiemas & \\
\hline & Programa de formalización de la propiedad rural & \\
\hline & \begin{tabular}{|l|} 
Adjudicación de tierras Incoder \\
Reestructuración del Programa (AIS) Agro Ingreso \\
Seguro y (DRE) Desarrollo Rural com Equidad \\
\end{tabular} & \\
\hline & Linea Especial de Crédito (LEC) & \\
\hline & Incentivo a la Capitalización Rural - ICR & \\
\hline & Programas de Asistencia Técnica & \\
\hline & Programa Formación en Extensión Rural & \\
\hline & Drenage) & \\
\hline & Programa Oportunidades Rurales & \\
\hline & Proyecto Apoyo a Alianzas Productivas & \\
\hline & Programa Mujer Rural & \\
\hline & Emprendimientos productivos & \\
\hline & Formación. & \\
\hline & Proyecto transversalización de género. & \\
\hline & Programa Jovenes Rurales & \\
\hline & Programa de jóvenes rurales-Becas ICETEX & \\
\hline & Emprendimiento productivo. & \\
\hline & Jóvenes de Educación Me dia. & \\
\hline & Alianzas & \\
\hline & Capacidades técnicas a pescadores & \\
\hline & Programa de Vivienda de Interés Social Rural & \\
\hline \multirow{12}{*}{ 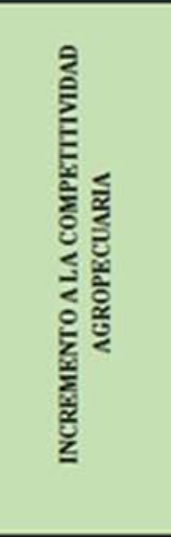 } & Credito agropecuario & \multirow{12}{*}{$\begin{array}{l}\text { Comición Nacional de Credito A gropecuario } \\
\text { (CNCA) } \\
\text { Fondo Nacional de Solidaridad A gropecuaria } \\
\text { (FONSA) } \\
\text { Ministeria de Agricultura y Desarrollo Rural } \\
\text { (MADR) }\end{array}$} \\
\hline & $\begin{array}{l}\text { Fortalecimiento del sistema financiero del sector } \\
\text { rural }\end{array}$ & \\
\hline & $\begin{array}{l}\text { Apoyo a la inversion rural atraves de } \\
\text { modificaciones al ICR }\end{array}$ & \\
\hline & Estrategia para asegurar la solvencia del FINAGRO & \\
\hline & Infraestructura de Riego y Drenage & \\
\hline & $\begin{array}{l}\text { Gestión de la pripiedad de distritos de adecuación } \\
\text { de tierras }\end{array}$ & \\
\hline & Investigación y desarrollo tecnologico & \\
\hline & Fortalecimiento sanitanio & \\
\hline & Programa de identificación trazabilidad animal & \\
\hline & programas de competitividad sectorial & \\
\hline & Plan de Acción de Reforestación Comercial & \\
\hline & Compromisos Paro Agranio & \\
\hline
\end{tabular}

Fuente: Recopilado de algunos de los principales programas dentro de las políticas para la agricultura familiar y el sector rural. Informe de rendición pública de cuentas. Gestión 2013 - 2014 


\section{Impactos generales de las políticas y el papel de la agricultura familiar en el desarrollo rural}

Maletta (2011), considera que las políticas públicas en Colombia enfocadas en el sector agropecuario y rural han presentado cambios en un periodo considerablemente relativo. Conforme el autor antes de los años 90, las instituciones funcionaban dentro de un esquema centralizado en el que a estructura de incentivos y medidas de protección tenían una influencia en los grupos de interés privados y políticos. Este tipo de esquema otorgaba mínimos incentivos a la participación y controle de los beneficiarios dentro de las políticas sectoriales. Adicionalmente con la apertura económica después de los años 90 se pretendió eliminar todas las distorsiones dentro de un esquema de protección selectiva e incentivos desviados, no se modificaron los hábitos ni las capacidades institucionales por lo que la modernización de la institucionalidad quedó incompleta. De tal forma que no generaron ningún tipo de impacto ya que las reformas fueron aplicados por muy poco tiempo y de manera marginal, desencadenando así en resultados heterogéneos para el sector y fracasos en sus instituciones.

En los últimos anos el desarrollo rural estaba asociado a expansión, diversificación, modernización de la producción, la comercialización de los productos agrícolas y las acciones de política que eran consideradas como las que conducían a esos propósitos. Según Perfetti (2013) en la actualidad se juzga sin suficientes fundamentos por dos razones principales, la primera porque crecimiento de la producción no es condición suficiente para alcanzar el desarrollo rural, y en segunda medida porque con los cambios de los últimos años, la producción rural no es sólo la agrícola sino que incluye diversidad de actividades. Se considera limitante el valor de la producción agrícola como variable para medir el desarrollo, cuando ese fenómeno, dentro del comando que las sociedades tienen sobre su entorno ya sea económico, social o ambiental. Perfetti (2013)

Siguiendo en la misma orden de ideas el desarrollo social se concibe como el resultado del crecimiento económico, pero también como un proceso de cambio que ultrapasa lo económico para llegar a otros aspectos de la vida social. Así el desarrollo rural debe buscar como resultado un crecimiento que promueva las igualdades sociales y con esto una distribución más equitativa de todos sus ingresos, que evite a toda costa la sobreexplotación de los recursos naturales y prueba la sustentabilidad ambiental. Además que facilite a acumulación de capital humano para fortalecer y potencializar las habilidades productivas y las capacidades para las zonas rurales, en donde se promueva una democracia y participación más eficaz que contribuya con el pleno aprovechamiento de los derechos humanos, que se propicie mejores condiciones de acumulación de capital y condiciones necesarias para fortalecer cuando los fines estén en peligro. Perfetti (2013)

Como se dejó ver en algunos apartados de este texto, no ha existido en Colombia una política clara y especifica de agricultura familiar, más existen políticas que se pueden relacionar, se trata de políticas del MADR y otras del Departamento Administrativo para la Prosperidad Social, que es una entidad del Gobierno Nacional que se encuentra dirigida por el Sector de Inclusión Social y Reconciliación. Por eso se hace tan importante el papel que representa la agricultura en el desarrollo rural.

Otra preocupación y cuestionamiento es que no se evidencias estudios académicos o por parte del gobierno que sean específicos en cuanto a la evaluación de los impactos que generan las políticas sobre la agricultura familiar o el pequeño productor y sus proyecciones a lo largo del tiempo. Pero por otro lado hay algunas investigaciones y trabajos de estudiosos o interesados en el tema que tratan de alguna manera sobre los efectos de las políticas de agricultura y desarrollo rural en Colombia. Se percibe también que las políticas han sido orientadas a favor de un modelo productivo empresarial, dejando de un lado a los sectores que más necesitan, que son los más pequeños y de menos recursos de la sociedad rural, políticas de tipo social que no llevan a un desarrollo ni a explorar sus recursos para mejorar sus condiciones. 


\section{Conclusiones}

Se requiere de un trabajo más arduo, en donde existan políticas diferenciales que acaben con las desigualdades socio-culturales e económicas. Una cuestión que no queda muy clara es, en que se han basado para estructurar las políticas rurales del país, si el último censo agropecuario ocurrió hace 45 años, es necesario re-valorizar los discursos sobre las personas del campo. Se hace necesario que las políticas para el fortalecimiento de la agricultura familiar tienen que partir de un esquema integral, sistematizado y con una proyección de largo plazo, para que exista continuidad en los procesos y se pueda evaluar con más cuidado y dedicación cada programa, política y acción del gobierno para fortalecer las condiciones no solo económicas, sino también sociales, ambientales y culturales.

Por lo tanto es necesario y fundamental los debates que se están comenzando a construir con diferentes instituciones, estudiosos e interesados en el tema, esto significa una importante contribución para el país, tener diferentes puntos de vista y formas de analizar las políticas que se han venido construyendo y las futuras, pensando en un modelo más integral, en donde todos salgan beneficiados desde el pequeño productor hasta el empresario. Es necesario políticas claras que manifiesten y tengan continuidad en sus procesos, no que sean fragmentadas y momentáneas, en donde no se pueda llevar continuidad y se tenga una evaluación más precisa de lo que ha generado y los pro y contras que eso conlleva.

El papel que representa la agricultura en el desarrollo rural es fundamental, es necesario partir desde un sistema de estadística más completo que abarque todas las dimensiones en las que están expuestos a populación rural, de esta forma se va poder identificar con exactitud a los pequeños productores, pero también es importante dentro de la lógica del mismo tema construir el concepto como una categoría de agricultura familiar para que de esta forma de consolide una definición para el país.

Por consiguiente se van a poder generar políticas diferenciadas por territorio, populación, donde exista una clasificación mejor para encaminar adecuadamente, políticas que respondan a las demandas que los territorios requieren, como indica Machado y Botello (2013), y que a su vez se tenga en cuenta la diferencia que existe entre economías campesinas que viven de la agricultura, y otras como las que tienen activos suficientes o insuficientes, las que cuentan con organizaciones sólidas, las que están en tierras de buena o mala calidad, , las que cultivan productos permanentes o transitorios y las que tienen condiciones competitivas y de otro tipo, se hace fundamental tener presente todos aquellos aspectos que van a beneficiar o perjudicar el sector rural, por eso que es tan importante cada elemento dentro de la misma dinámica. Resaltó con lo anteriormente mencionado que diferentes países de América Latina e instituciones como es el caso de la FAO han reconocido la importancia de la agricultura familiar y de los pequeños productores dentro del desarrollo rural y sustentable de cada país.

\section{BIBLIOGRAFÍA}

ALPHA, A. y CASTELLANET, C. Défendre les agricultures familiales: lesquelles, pourquoi?. Résultats des travaux et du séminaire organisé par la Commission Agriculture et Alimentation de Coordination SUD., 2008.

CAMPOS, A. P., GARNER, Elizabeth. Defining the "Family Farm". Working paper. FAO, p. $29,2012$.

CRECE. Centro de Estudios Regionales Cafeteros y Empresariales. 2006. "Pobreza rural: diagnóstico y evaluación de las políticas nacionales”. Manizales, Colombia.

Gómez H., B. 2011. La tenencia de la tierra y la reforma agraria en Colombia. Verba Iuris. 63-83.

Giarracca Norma. 2001. ¿Una nueva ruralidad en américa latina. Consejo Latinoamericano de Ciencias Sociales / CLACSO

Gutiérrez López, J. A. 2009. La estadística estratégica del sector agropecu ario em Colombia: un nuevo modelo de oferta. (D. -D. Estadística, Ed.) Ib Revista de la información básica, http://www.dane.gov.co/revista_ib/html_r6/articulo3_r6.html.

HERNÁNDEZ, Roberto. Teorías sobre campesinado en América Latina: Una evaluación crítica. Revista Chilena de Antropología, n. 12, 1993. 
Ministro de Agricultura y Desarrollo Rural. Colombia 2014. Informe de rendición pública de cuentas. Gestión 2013 2014.

Machado C., A. 2009. Ensayos para la historia de la política de tierras en Colombia: de la colonia a la creación del Frente Nacional. Universidad Nacional de Colombia., Facultad de Ciencias Económicas. Centro de Investigaciones para el Desarrollo, CID. Bogotá: Universidad Nacional de Colombia. Facultad de Ciencias Económicas. Centro de Investigaciones para el Desarrollo, CID.

Machado C., A., \& Botello M., S. 2013. La Agricultura Familiar En Colombia (Manuscrito no publicado). RIMISP-FIDA.

Machado, A., Salgado, C., \& Naranjo, S. 2013. Territorios para el desarrollo de las sociedades y economías campesinas. En Oxfam, Reflexiones sobre la ruralidad y el territorio en Colombia (págs. 275-366). Bogotá: Oxfam.

Malagón, D. 2002. "Los suelos de Colombia”. Revista de la academia de ciencias geográficas, 46, (135).

Maletta, H. 2011. Tendencias y perspectivas de la agricultura familiar en América Latina. Santiago, Chile: Documento de Trabajo $N^{\circ}$ 1. Proyecto Conocimiento y Cambio en Pobreza Rural y Desarrollo. Rimisp.

Perfetti, J.J. 2009. "Crisis y pobreza rural en América Latina: El caso de Colombia". Documento de trabajo No 43, Programa Dinámicas Territoriales Rurales, Rimisp, Santiago, Chile.

Perfetti, J. J., \& Cortés, S. 2013. La agricultura y el desarrollo de los territorios rurales. En J. J. Perfetti, Á. Balcázar, A. Hernández, \& J. Leibovich, Políticas para el desarrollo de la agricultura em Colombia (págs. 1-64). Bogotá: Sociedad de Agricultores de Colombia (SAC) y Fedesarrollo.

Sabourin. E., Samper. M., \& Sotomayor, O. 2014 Políticas públicas y agriculturas familiares en América Latina y el Caribe. Balance, desafíos y perspectivas. Comisión Económica para América Latina y el Caribe (CEPAL)

SANCHES P., A. Agricultura familiar: Evolución conceptual, desafíos e institucionalidad. FAO - Iniciativa América Latina y Caribe Sin Hambre 2025, Lima. 2011.

SALCEDO S.; G., Lya. Agricultura familiar en américa latina y el caribe: recomendaciones de políticas. FAO. Santiago de Chile, 2014.

Vélez. A., Campos, A., Córdoba, S. \& Anzola, J.F. 2010. "Invierta en Colombia: Sector agroindustrial colombiano””. Vol I.

ZANI, F. B.; DA COSTA, F. L. Avaliação da implementação do Programa Nacional de Fortalecimento da Agricultura Familiar—novas perspectivas de análise. Revista de Administração Pública, v. 48, n. 4, p. 889-912, 2014. 